\title{
Radicalités et modérations, postures, modèles, théories
}

Naissance du cadre politique contemporain

\section{Pierre Serna}

\section{(2) OpenEdition \\ Journals}

\section{Édition électronique}

URL : https://journals.openedition.org/ahrf/10527

DOI : 10.4000/ahrf.10527

ISSN : 1952-403X

Éditeur :

Armand Colin, Société des études robespierristes

Édition imprimée

Date de publication : 1 septembre 2009

Pagination : 3-19

ISBN : 978-2-200-92559-8

ISSN : 0003-4436

\section{Référence électronique}

Pierre Serna, « Radicalités et modérations, postures, modèles, théories ». Annales historiques de la Révolution française [En ligne], 357 | juillet-septembre 2009, mis en ligne le 09 décembre 2009, consulté le 22 avril 2022. URL : http://journals.openedition.org/ahrf/10527 ; DOI : https://doi.org/ 10.4000/ahrf. 10527 


\section{RADICALITÉS ET MODÉRATIONS, POSTURES, MODĖLES, THÉORIES. NAISSANCE DU CADRE POLITIQUE CONTEMPORAIN ${ }^{1}$}

Pierre SERNA

\section{La radicalité à l'ordre du jour ?}

En soi, la Révolution est radicalité, rupture nécessairement violente, pensée, et vécue comme telle ${ }^{2}$. Le hors norme, l'exagération s'imposent à tous, dans l'immédiateté de la prise de conscience de ce qui se passe, dès le printemps 1789. Quelque chose se produit qui dépasse 1'entendement. Une série d'événements inattendus a exigé une énergie, une mise en jeu des forces, inventant une dynamique qui repousse toute idée de modération, de prudence, vers le passé, rapidement constitué en Ancien, puis en Ancien Régime qui ne sera plus. Et avec lui le cortège de ses représentations, parmi elles sa douceur, sa tempérance, rapidement érigées en souvenirs nostalgiques... par une minorité qui seule pouvait y goûter.

Dans les correspondances privées, là où les âmes s'épanchent plus volontiers, surgissent aux détours des confidences, les marques des évé-

(1) Si le mot de modération est bien connu des contemporains et utilisé dans le champ politique, signalons que l'usage du mot radicalité pour la période révolutionnaire est quelque peu anachronique puisqu'il apparaît dans son sens politique en 1820, d'un emprunt fait à la langue anglaise, cf. Alain REY (dir.), Dictionnaire historique de la langue française, Paris, Éd. Robert, 1992, t. II, p. 1702. À l'époque, en revanche, le terme « exagéré » est le plus souvent utilisé pour exprimer cette radicalité politique.

(2) ... et 220 ans après, le paradigme est encore efficace. Dans un article publié dans Le Monde daté du jeudi 16 avril 2009 sous le titre « Les acteurs politiques face au piège de la radicalité », Françoise Fressoz conclut ainsi : « On se croirait sous l'Ancien Régime, constate Ségolène Royal, tandis que Nicolas Sarkozy, se souvient que les Français ont coupé la tête au roi ». 
nements qui scandent les mois de mai, juin, juillet, août 1789, que les hommes à Versailles essaient de traduire dans leurs mots, lorsqu'ils écrivent à leurs épouses, à leurs régisseurs, cherchant dans leur vocabulaire convenu les marques de ce saut dans l'irréel radical de la révolution en marche ${ }^{3}$. Points d'exclamations, adjectifs admiratifs ou inquiets, aveux de la faiblesse des mots ( je ne saurai vous dire... »), marquent la limite d'un vocabulaire convenu et déjà insuffisant pour exprimer le réel bouleversé. À la fin de l'année 1789 , plus de 300 journaux ont vu le jour. La libération de la parole offre un miroir à l'ampleur des transformations en cours : dans un jeu de fondu-enchaîné, les écrits décrivent le réel, lui donnent une épaisseur réflexive qui à son tour, dans la réalisation de l'inimaginable de la veille permet toujours plus d'audace intellectuelle. Dans cette société française soudainement libre, dire c'est faire, et faire c'est déjà provoquer le dire ${ }^{4}$. Le verbe est performatif, le faire est déjà discours, double performance qui donne au réel, dès le mois de juillet 1789 , une dimension de surréalité qui induit forcément le sentiment d'une existence et d'une pensée ayant basculé dans une radicalité, jusque là inconnue.

La dynamique enclenchée, rien ne semble plus l'arrêter, et ce ne sont pas les événements des étés 1791, 1792, 1793 puis de 1794 qui vont permettre de laisser imaginer qu'une quelconque tempérance et modération pourraient s'imposer à une mécanique politique qui semble s'autonomiser dans une surenchère impossible à arrêter. La révolution-machineemballée devient la figure même d'une radicalité à l'œuvres. Et puis l'été 1794, l'an III et son automne, puis le printemps 1795 avec la poursuite de la répression du mouvement ouvrier, largement commencée au printemps de l'an II, la république des gens de bien, des personnes aspirant à la tranquillité de l'ordre public, la prégnance d'un modèle bourgeois fondé sur les vertus discrètes du savoir, de l'action silencieuse, de la distinction tranquille, de la désidéologisation de la société, source de querelles, de désordres, de radicalité intempestive. 1795 ou le début de l'endormissement et de la modération forcée ? L'épisode se terminerait avec l'homme

(3) Jean-Pierre Jessenne, Edna Hindie Lemay, avec l'assistance de Martine Aubry, Député paysan et fermière de Flandre en 1789. La Correspondance des Lepoutre, Lille, Centre d'histoire de 1'Europe de Nord-Ouest, 1998, et Timothy Tackett, Par la volonté du Peuple. Comment les députés de 1789 sont devenus révolutionnaires, Paris, Albin Michel, 1996.

(4) Claude Labrosse, Pierre RÉTAt, Naissance du journal révolutionnaire, 1789, Lyon, Presses universitaires de Lyon, 1989.

(5) Michel Biard, Parlez-vous sans-culotte? Dictionnaire du Père Duchesne (1790-1794), Paris, Tallandier, 2009. 
providentiel « ni bonnet rouge, ni talon rouge », venu terminer la Révolution en apportant la paix des cours, des esprits et des braves.... C'est à voir.

Las, quelque chose n'a pas dû fonctionner... et ce n'est pas le général corse qui inspire le mieux dans l'histoire de France l'image du souverain le plus calme, le plus posé... Rien, en effet, ne serait plus réducteur d'imaginer l'après 1794 , comme la période de calme bourgeois, ayant brisé irrémédiablement la dynamique portée par les années précédentes, par des couches populaires «naturellement 》 portées aux extrémités. Procédant ainsi, bien des historiens se faisant naturellement les avocats d'un peuple actif, renforçaient l'édification de catégories, et à leurs corps défendant apportaient moult arguments à d'autres historiens trop heureux de lire la confirmation de la normale agitation du peuple comme trait distinctif de son incapacité génétique à parvenir au calme, à la raison nécessaire à la conduite de la chose publique. Il faudrait plutôt convenir qu'après 1794 , la radicalité s'est déplacée sur d'autres espaces, à la guerre, par exemple, provoquant l'étincelle qui va embraser toute l'Europe du XIX siècle, inventant l'État-nation comme l'essence même d'une radicalité belliqueuse à la potentialité dévastatrice. La radicalité s'est aussi transportée dans l'espace des idées, introduisant la liberté et l'égalité au centre des débats, à la gauche de l'échiquier politique, inventant le babouvisme, puis la théorie de la démocratie représentative parmi les républicains démocrates du Directoire, berceaux théoriques et bientôt moteurs pratiques des révoltes patriotiques dans les Républiques-sœurs. N'y a-t-il pas dans ces deux formes politiques, le complot ou la conquête du suffrage le plus large, l'essence des révolutions du XIX siècle, lorsque ces deux matrices se seront transformées dans les idéaux radicaux du socialisme ou du communisme naissant?

La droite politique ne saurait rester indifférente à ces formes énergiques de l'action et de la pensée politique. Elle aussi connaît, par le biais de l'expérience révolutionnaire traumatisante, une radicalisation que la modernité politique de la fin du XVIII ${ }^{\mathrm{e}}$ siècle va lui conférer. Épurée par la flamme de l'adversité, la pensée dite ultra, n'a jamais cessé d'exister, confortée par les échecs de la révolution ${ }^{6}$. Les avocats de la monarchie d'origine divine voient dans les épreuves le sens même d'une régénération

(6) Jean-Clément Martin, Contre-Révolution, Révolution et Nation, en France 1789-1799, Paris, Seuil, 1998. 
qui doit valider leur dévotion à un pouvoir absolu, fondé sur le mystère de la foi catholique, vécue dans la radicalité de la soumission aux autorités temporelles, et le don total de soi à l'autorité du principe divin. Une autre famille de droite trouve un biais de radicalisation, non dans une idéologie de la Restauration, mais dans la sociologie politique de la Contre-Révolution, dans un apport a priori inespéré, celui d'une partie d'un peuple, soudé dans un anti-révolutionnarisme ne révélant nullement son indécrottable archaïsme, ni son passéisme atavique, mais sa claire compréhension des nouveaux modes de domination urbains et bourgeois que la Révolution a créés, confisquant au plus grand nombre une émancipation espérée. Pensée contre-révolutionnaire, mobilisation des pratiques populaires antirévolutionnaires, esquissent du côté droit de l'échiquier une radicalisation qui n'a rien à envier à l'autre bord politique.

Tout serait dit et la radicalisation serait un mal français apporté à la modernité. La tare de l'exagération et de la surenchère constituerait une marque de fabrique typique de la politique telle que la décennie 17891799 l'aurait laissée en héritage empoisonné au futur. Les élites du pays seraient victimes de leur emphase, toute calquée sur une Rome de papier, sortie de la lecture au premier degré de Plutarque, découvert dans les collèges de la monarchie. Le peuple, lui, serait le jouet de ces pulsions sanglantes et populairement gauloises. Pire même, les conséquences pratiques de ces postures rhétoriques, voire politiciennes, auraient anticipé, voire rendu possible la violence bien réelle des guerres civiles, fratricides et meurtrières que la France allait connaître de façon endémique, jusqu'à la Libération, après $1945^{7}$. À force de se prendre pour des Romains, les Français seraient devenus des barbares...

Sûrement faut-il nuancer cette impression construite en vérité historique, et relancée comme une certitude stigmatisante par chaque génération de détracteurs de 1789 et de ses conséquences. De nos jours une historiographie dominante dans ce qu'il est convenu d'appeler le « grand public », s'est édifiée à l'époque des dictatures communistes en Chine, en Union Soviétique et au Cambodge, se démarquant du marxisme et cherchant à inventer une gauche pleinement réconciliée avec l'économie de marché, une gauche recherchant une tempérance idéologique au profit d'une efficacité économique, après les chocs sociétaux de 1968 et pétrolier de 1974. Trente ans plus tard, plusieurs historiens ont grandement

(7) Anne Simonin, Le déshonneur dans la République : une histoire de l'indignité 17911958, Paris, B. Grasset, 2008. 
contribué à nuancer cette perception tranchée du fait révolutionnaire en marche, en portant leurs regards sur les origines lointaines et monarchiques de la radicalité, et en élargissant leurs regards vers d'autres espaces géographiques.

\section{Changer de cadre chronologique, élargir l'horizon géographique}

La question de la radicalisation, sous toutes ses formes, travaille la modernité depuis le $\mathrm{XVI}^{\mathrm{e}}$ siècle florentin et la théorisation "savonarolienne » de la souveraineté populaire, ou machiavélienne de la souveraineté du prince. Les pères fondateurs des sciences politiques, Hobbes, Bodin, entre autres, n'ont eu de cesse de penser les expressions de l'Ubris qui menacent en permanence les constructions monarchiques entièrement repensées durant la crise de la Réforme, puis des guerres de religion ${ }^{8}$. Tous savent que la radicalité, conçue comme l'expression d'une borne franchie, la rupture d'une norme dans l'exagération arbitraire d'un pouvoir, d'une prérogative légale ou légitime, ne vient pas tant du «peuple» largement inexistant comme force politique fédérée, que du prince lui-même et de sa pente naturelle à s'octroyer toujours plus de pouvoir. Encore faut-il nuancer de suite cette assertion, en traversant par exemple la Manche et en rappelant succinctement ce qu'une historiographie Whig a copieusement caché avant que Christopher Hill et son école ne retrouvent l'authentique violence et radicalité qui avaient précédé la Glorieuse et présentable Révolution de $1688^{\circ}$. Bien avant les Français, et dans une perspective qui dépasse le seul cadre religieux des luttes du siècle précédent, les nivellers anglais vont assumer une rupture totale avec les cadres de la politique ancienne. Portés par le mouvement de révolte qui mènera à l'exécution de Charles ${ }^{\mathrm{er}}$, en 1649, ils vont décider de traduire politiquement l'enseignement des évan-

(8) En un Ancien Régime où religion et politique se trouvent mêlées, nombreux sont les observateurs qui remarquent combien la religion est en soi facteur de radicalité clairement perçu, souvent sous le terme de fanatisme, et comment la politique, justement menée, peut devenir au contraire un facteur d'apaisement des tensions par la laïcisation des enjeux et l'invention d'un bien commun public. Cf. Olivier Christin, La paix de religion. L'autonomisation de la Raison politique au $X V I^{e}$ siècle, Paris, Seuil, 1997.

(9) Christopher Hill, Le monde à l'envers. Les idées radicales au cours de la Révolution anglaise, Paris, Payot, 1977 (1972 pour la première édition). Je ne méconnais pas les travaux de Jonathan Israël, Les lumières radicales. La philosophie, Spinoza et la naissance de la modernité, Paris, Éditions Amsterdam, 2005 (2001 1 1e éd.), mais la référence anglaise est certainement plus explicite au début de la Révolution que l'allusion spinozienne. $C f$. Antoine Lilti, « Comment écrit-on l'histoire intellectuelle des lumières ? Spinozisme, radicalisme et philosophie », Annales HSS, janvier-février 2009, n 1 , p. 171-206. 
giles au risque de bouleverser l'ordre social, de mettre le monde le «cul par-dessus tête ». Réprimés, pourchassés, exécutés, exilés, poursuivis, ils avaient largement disparu du paysage historiographique, condamnés par leur radicalité même, transposition utopique d'un autre monde meilleur, ici-bas. Las, c'était sans compter sur la ténacité de l'historien Marcus Rediker qui, récemment, poursuivant ces travaux, a montré combien cette « hydre aux mille têtes », celle de la mauvaise graine de tous les hors-la-loi intransigeants, criminalisés au nom de leurs égalitarisme, avait continué d'exister, jusqu'au début du XVIII siècle, semant la terreur aux abords des Caraïbes, toujours prête à se mutiner dans l'entrepont cauchemardesque des bâtiments de la Royal Navy ${ }^{10}$. Les chartes de pirate, le sens politique que Daniel Defoe, sous couvert d'un faux nom, déchiffre dans ces républiques d'hommes sanglants mais partageurs, disent les formes complexes de la radicalité, ses exils, ses tours et détours, aux marges antillaises du monde européen dit civilisé ${ }^{11}$. Il a fallu au début du XVIII ${ }^{e}$ siècle, l'Espagne d'après le traité d'Utrecht n'étant plus un danger pour la City, toute la volonté féroce de Londres pour éradiquer et détruire la canaille pirate, pour étouffer un temps une des expressions les plus fortes et les moins reconnues du point de vue politique de la sédition radicale et populaire ${ }^{12}$. Les formes les plus sévères de l'exercice de la justice par le spectacle constant et public des supplices finaux sur les quais de Londres se trouvent au cœur de cette éradication d'un extrémisme populaire. Le spectacle de l'exécution avant et après la Révolution constitue un lieu important d'une réponse possible de l'ordre public à ce qu'il perçoit toujours comme un désordre ou la rupture d'une règle mettant en danger les formes de fonctionnement de la cité. La justice du roi invente une réponse qui malgré sa violence se veut toujours réponse mesurée ${ }^{13}$. Ainsi, le juste et l'injuste se trouvent au cœur d'une réflexion sur le radical et le modéré. La scène initiale de Surveiller et punir de Michel Foucault racontant en détail l'exécution de Damiens en mars 1757 , met en scène non la radicalité mais la réparation voulue comme juste et présentée dans la suite des supplices infligés au régicide et ce faisant, permet de mesurer un décalage avec une nouvelle sensibilité née du rejet de

(10) Markus Rediker et Peter Linebaugh, L'hydre aux mille têtes, l'histoire cachée de l'atlantique révolutionnaire, Paris, Éditions Amsterdam, 2008 (2001 pour la première édition).

(11) Daniel Defoe, Histoire générale des plus fameux pirates, Paris, Payot 1992 (2 vol.), 1724-1726 pour les premières éditions.

(12) Pierre Serna, «Libertalia ! Debout les damnés de la mer », dans Michel Le Bris et Virginie Serna (dir), Pirates et flibustiers des Caraïbes, Éd. Hoëbeke, 2001, p. 178-189.

(13) Pascal Bastien, L'exécution publique à Paris au XVIII siècle. Une histoire des rituels judiciaires, Seyssel, Champ Vallon, 2006. 
cette violence légale, perçue par un public éclairé (et jusqu'au roi lui-même) comme l'expression même de l'abus du pouvoir et de sa justice exécutante disproportionnée ${ }^{14}$.

\section{La monarchie absolue ou les origines politiques de la Révolution}

Voilà aussi pourquoi très vite, les théoriciens du politique avant 1789 ont pensé la radicalité, point tant autour du dire et des idées législatives que du faire et de l'acte exécutif, se référant à l'importance de la parution en 1748 de De l'esprit des lois de Montesquieu. Désormais, grâce au magistrat bordelais, le problème des sciences politiques n'était plus tant de penser les conditions de contrôle du monarque hérité du système de Louis XIV, que de construire une politique fondée sur la raison, la mesure et la tempérance. Le nouveau régime qu'imagine Montesquieu doit reposer sur la modération, non comme un frein, mais comme un principe actif, soit par la séparation des pouvoirs, soit par leur division, soit par leur équilibre, en tout cas dans leur co-action modérée ${ }^{15}$. La modération comme force révolutionnaire, c'est-à-dire comme pensée capable de déstabiliser les fondements de la monarchie absolue est née, et va structurer l'espace de l'opinion et de l'échange publics qui connaît un premier apogée entre 1750 et 1770 . La raison critique mesurée, démocratisée, débattue devient un modèle de gouvernement. Elle défend la construction d'un vivre ensemble dont les tensions internes se trouvent contrôlées et régulées par une Constitution, par une représentation, par une liberté d'expression qui finissent par produire un système politique organisé autour de la modération, et la liberté, que l'Angleterre le plus souvent mythifiée, vient illustrer. « La raison est un juste milieu, à l'opposé des extrêmes » ${ }^{16}$.

(14) Michel Foucault, Surveiller et punir, Paris, NRF/ Gallimard, 1975.

(15) Ran Halevi, « La modération à l'épreuve de l'absolutisme. De l'Ancien régime à la Révolution », Le Débat, mars-avril 2000. Céline SPECTOR, Montesquieu et l'émergence de l'économie politique, Paris, Honoré Champion, 2006.

(16) Les contemporains connaissent l'œuvre publiée en 1767 par Changeux Traité des Extrêmes, ou Éléments de la science de la réalité, dont le supplément de l'Encyclopédie de Diderot et d'Alembert, dirigé par Panckoucke rend compte. «L'avertissement ou plutôt la préface nous apprend, que l'auteur avait entrepris de faire, pour l'Encyclopédie, l'article RÉALITÉ ; [...] il ajoute qu'il commence par distinguer la réalité de la vérité, \& qu'il a cherché à découvrir le caractère de la réalité, de la même manière que Descartes avait découvert celui de la vérité ; qu'il a trouvé que le moyen de reconnaître la réalité était fondé sur un principe, d'où découlaient une foule de conséquences dans tous les genres de connaissances : il ajoute que la science de la réalité est plus dure que celle de la vérité, avec laquelle on ne pourra plus à l'avenir la confondre. Il dit : Dans la constitution présente de l'homme, les extrêmes se touchent sans se confondre, \& la réalité ne se trouve que dans le milieu qui est entre les deux extrêmes ». 
Face à ces critiques, la monarchie ne reste pas inactive, choisissant délibérément une autre voie, celle de la radicalisation possible et de son efficacité par le choix (raté) du despotisme éclairé ${ }^{17}$. À ce titre, les années 1766-1774 sont essentielles pour introduire le bouleversement politique de la génération suivante. Lorsque Louis XV vient tenir son Parlement, lors de la séance de la flagellation, le 3 mars 1766, se joue un sommet dramatique de l'opposition entre officiers magistrats et commissaires du roi. Le souverain brandit la menace explicite de se saisir de tous les pouvoirs qui se trouvent potentiellement en sa main pour imprimer la terreur (on l'a oublié) à ses sujets désobéissants. Quatre ans plus tard, en janvier 1770, Terray et Maupeou assument ce rôle dans le coup d'État légal qui bouleverse les lois fondamentales du royaume en conférant au roi un pouvoir disproportionné, radicalement autoritaire par rapport à l'esprit des institutions monarchiques dont il aurait dû être le défenseur. Il revient à Dale van Kley d'avoir pointé avec une rare finesse la rupture de ce moment qui voit les deux sphères janséniste et jésuite (les deux seules figures possibles d'expression du politique dans un espace royal qui n'en a pas) exploser en deux sous-ensembles ${ }^{18}$. L'opposition résolue qui les divisait en deux familles irréconciliables se nuance devant le coup royal et se subdivise en deux sous-groupes peu perceptibles mais essentiels pour comprendre l'ampleur de ce qui vient de se jouer. Les plus lucides des deux camps comprennent la gravité de la situation, décidant désormais de s'en tenir à une attitude modérée. Ils réalisent que le coup de majesté, loin de renforcer le pouvoir du roi, risque de le fragiliser en ces temps de montée en puissance de cette chose nouvelle qu'est l'opinion publique. Les modérés des deux camps en appellent déjà à la sagesse et au calme des humeurs. Les plus emportés des deux camps saisissent tout autant ce qui se joue mais décident de pousser ce qu'ils pensent être leur avantage. Les jansénistes les plus «à gauche » ne rêvent plus que d'États généraux. Les partisans des jésuites expulsés peu auparavant, les plus «à droite », n'espèrent plus qu'une fermeté encore plus grande du roi.

De fait, la radicalité avait de lourds antécédents lorsque la Révolution commence, précédents largement responsables, selon bien des observateurs de la dérive néfaste des institutions du royaume. Ce constat était essentiel à clarifier car il permet de renverser la proposition originelle

(17) Michel Antorne, Louis XV, Paris, Fayard, 1989.

(18) Dale K. VAn Kley, Les origines religieuses de la Révolution française, 1560-1791, Paris, Seuil, 1996. 
de l'introduction : la Révolution n'est pas une radicalisation quelconque et naturelle, par le saut dans un vide politique.

\section{Radicalité, modération avers et/ou envers d'un même champ politique?}

1789 est l'aboutissement d'une recherche désespérée de la part d'une génération pour inventer une modération politique à partir de la lecture de Montesquieu et de l'expérience négative des années qui vont de la défaite de la Guerre de Sept Ans en 1763 aux conséquences dramatiques du succès de la guerre d'indépendance des États-Unis en 1783 ! La Révolution, dans ces conditions, consiste en la tentative de sortir du caractère incontrôlable de la monarchie, de l'anarchie arbitraire du bon vouloir de sa majesté, de la radicalité possible de la couronne, comme le dernier coup de majesté en mai 1788 l'a démontré (ultime tentative de s'imposer illégalement à la magistrature), pour inventer une tempérance, une organisation rationnelle de la vie politique. Toute la difficulté pour l'historien consiste donc à ne pas se laisser emporter par la facilité avec laquelle on a tôt fait de comparer, " comme de bien entendu », la Révolution avec une ère de radicalisation évidente. Au contraire, sa tâche consiste à tenter de comprendre comment la période qui s'ouvre en mai 1789 amène à la recherche brouillonne, et parfois contradictoire d'un mode de gouvernement, de construction de la liberté de l'opinion publique et d'émancipation de la société qui puisse s'insérer dans un mode de régulation des tensions inhérentes à un régime de libertés, pour autant contrôlées par des lois, fixant les limites nécessaires à la modération. Cette dernière se résume à la co-gestion de l'espace où la liberté des uns est limitée par celle des autres ${ }^{19}$. Les contemporains eux-mêmes, avant de passer au tamis sévère de l'analyse historique devaient avoir bien du mal à se positionner et à prendre parti entre modération et radicalité. C'est qu'avant de penser le clivage entre les deux tendances puis les sous-ensembles à l'intérieur de chaque famille politique, les acteurs, dans leur for intérieur, pouvaient légitimement ressentir quelques difficultés au moment de se positionner.

(19) L'apport de Kant à cette réflexion est à souligner avec la claire perception des conflits de la politique moderne mettant face à face d'un côté un pouvoir toujours absolu et de l'autre l'avènement de la liberté imaginé par les Lumières dans un jeu des libertés affrontées et d'autant plus opposées qu'elles doivent s'exprimer librement, dans un espace public nouveau. Cf. KANT, Vers la paix perpétuelle, Présentation de François Proust, Paris, GF, 1991 (1795, première date de parution). 
Relisons Sade à ce sujet qui en perd son latin, au moment d'écrire à Gaufridy, qui lui tient lieu d'intendant en Provence le 28 décembre 1791 : « Je suis antijacobite, je les hais à mort, j'adore le roi, mais je déteste les anciens abus, j'aime une infinité d'articles de la constitution, d'autres me révoltent» et finit par conclure : "Que suis-je à présent ? Aristocrate? démocrate ? vous me le direz s'il vous plait, avocat, car pour moi, je n'en sais rien $»^{20}$. Modéré ou radical ? La question est authentiquement existentielle et encore plus pour le marquis, conscient de la puissance des mots et connaissant parfaitement le pouvoir des coups. Par bien des aspects, l'étude de l'engagement du divin marquis devenu révolutionnaire, illustre la difficulté d'une réflexion sur la frontière étanche entre radicalité et modération. Idées modérées, actes radicaux, idées radicales, actes modérés ? Comment concilier les contradictions ?... Être modéré dans un mouvement radical ou devenir radical dans un mouvement modéré, le tout décliné chaque année selon la conjoncture et le « vent tournant », n'est ce pas le destin dramatique de bien des contemporains du marquis ? Tout à coup les quelques certitudes de l'historien du début du XXI siècle se trouvent fragilisées. L'intérêt pour l'étude de la politique en révolution ne s'en trouve que relancé car complexifié, hors des cadres d'interprétation rigides, ni dans un relativisme anthropo-historique qui résume toute action et toute parole à une attitude politicienne, modération ou radicalité se neutralisant, ni dans une surdétermination idéologique soupçonnant dans chaque manifestation d'une personne la preuve de l'influence d'un cadre structurel à interroger plutôt que d'écouter ce que les personnes disent de leurs motivations au moment d'agir dans le cadre d'une modération assumée ou d'une radicalité pensée.

La parole et les actions de Robespierre d'un côté, mériteraient d'être réexaminées dans ce cadre. Lui qui fait figure d'une rigidité toute radicale, n'a de cesse de dénoncer les ultra et les citra-révolutionnaires comme les authentiques ennemis de la Révolution, tentant de chercher, de trouver une voie médiane pour sauver la République ${ }^{21}$. Robespierre n'est pas le seul, loin s'en faut, durant la décennie révolutionnaire à construire un système de dénigrement des radicaux en « extrémistes » risquant sans

(20) Cité dans Gilbert Lely, Vie du marquis de Sade, Paris, J.J. Pauvert éditions, 1982, p. $452-453$.

(21) Cf. Robespierre, Euvres complètes, discours du 5 nivôse an II, et discours non prononcé sur la faction de Fabre d'Eglantine, fin nivôse an II, p. 312-315 et 326-335. Voir également l'analyse proposée par Albert SoBoul, Histoire de la Révolution française, Paris, Gallimard, 1962, tome I, De la Montagne à Brumaire, p. 65-68. 
cesse de rompre le fragile équilibre de la construction politique. D'un autre côté, le destin tragique d'un Camille Desmoulins, radicalement à la gauche de l'échiquier en 1789, se retrouvant sans avoir changé, dans le camp des «modérateurs » de la Terreur cinq ans plus tard en 1794, et le payant de sa vie peut permettre de comprendre aussi les stratégies personnelles de ceux qui, sentant le vent tourner au fur et à mesure de la décennie, se sont adaptés selon des conjonctures différentes à des postures différentes, prudents en temps de réaction, ardents en temps d'accélération du processus, contribuant fortement à décrédibiliser, sitôt née, la politisation en acte. Robert Palmer mais aussi Richard Cobb ne voudront percevoir dans ces comportements que la lie d'une classe politique indigne mais au fond révélatrice de l'ensemble d'une société française suiviste et imparfaitement intégrée au processus révolutionnaire. Au lieu de régénérer une population rendue servile par la monarchie, cette élite corrompue avait révélé ses pires défauts d'avidité, d'égoïsme que le transformisme et la violence politique mettaient à nu sans fard et de la façon la plus vile qui soit dans la volte face des radicaux Tallien, Fréron, Barras, agents de la Terreur, subitement métamorphosés en réacteurs tout autant radicaux, ou en modérés à la façon de 1'an III ${ }^{22}$. Le cadre inconstant, fragile, soumis aux brusques changements des aléas des crises rend difficile la définition stricte des mots et le repérage sûr de chacun des acteurs sur la scène politique ${ }^{23}$. L'historien se doit d'adopter à son tour une souplesse interprétative à moins de plaquer ses propres « radicalités » ou «modérations » sur les positionnements anciens.

Dans cette perspective, le parcours d'un Louis-Sébastien Mercier est fort instructif. L'homme d'avant 1789, celui du Tableau de Paris, se veut raisonnable, adversaire de tous les fanatismes qu'il n'a de cesse de dénoncer, de moquer en en décrivant les aspects dangereux pour le bien vivre ensemble. Tous ceux qui sont enfermés dans un système rigide de pensée, incapables d'intégrer une autre pensée que la leur, dans l'impossibilité d'évoluer ou de changer au moment d'écouter les arguments raisonnés de leurs contradicteurs, sont désignés comme des «fanatiques », mettant en péril la cohésion sociale. Dévots, jansénistes tristes, jésuites ardents, tout y passe pour dénoncer un système où les extrêmes seraient

(22) Robert Palmer, Le gouvernement de la Terreur, Paris, Armand Colin, 1989 (I ${ }^{\mathrm{re}}$ éd. 1941, Twelve who ruled) et Richard Cовв, La protestation populaire en France (1789-1820), Paris, Calmann-Lévy, 1975 (1970, Ire éd.).

(23) Sergio Luzzatto, L'automne de la révolution, luttes et cultures politiques dans la France thermidorienne, Paris, Champion éditeur, 2000 (1994, Ire éd.) 
reliés secrètement, se « toucheraient », liés par un pacte de destruction de l'ordre existant ${ }^{24}$. L'homme de lettres qui sort de prison, après l'épisode de la Terreur se met à rédiger son Nouveau Paris durant le Directoire. Les conditions politiques ont bien changé. Profondément choqué par l'expérience de la radicalisation populaire et parisienne dont il a eu à souffrir en tant que député proche de la Gironde, l'observateur des mœurs de la capitale maintient son cap cherchant dans les voies de la régulation et de la modération, l'unique possibilité de sortie de la crise permanente dans laquelle s'est enfoncée la République, acceptée comme unique voie pour terminer la Révolution. Cette fois-ci, dix ans après la parution du Tableau de Paris, la révolution tant attendue et espérée pour mettre fin à l'arbitraire royal est devenue synonyme de violence exacerbée et de radicalisation incontrôlée. Mercier dénonce certaines élites irresponsables et soupçonnées d'attiser la logorrhée la plus extrémiste pour se maintenir au pouvoir réduisant la surenchère à une posture politicienne. La Révolution est devenue également synonyme de désordres dès lors qu'une portion de la « populace » (terme générique aisé à utiliser dans le discrédit flou et précis à la fois qu'il induit), ignorante et naturellement portée à la sédition, participe au jeu trop compliqué pour elle de la science politique requérant savoir, expérience, calme, concertation, voire compromis, en un mot modération par le biais de la participation exclusive des « meilleurs », les propriétaires, à la vie de la cité.

\section{La modération par le centre, ou l'abus de pouvoir permanent}

Il faut reconnaître que la nouvelle donne politique, issue de 1795, a brouillé tous les termes et a inventé un nouveau vocabulaire politique, lorsqu'elle n'a pas transformé le sens même des mots, changeant de fait l'interprétation des faits et gestes. Mercier en personne écrit à la fin de la période un dictionnaire des néologismes pour s'y retrouver ${ }^{25}$. Certes, Thermidor hérite d'un champ qui peut être divisé en trois entités, composées des radicaux de gauche et de ceux de droite qui ne tardent pas à reparaître, séparés par le marais, centre difficilement cernable et renaissant sans cesse de façon différente. Déjà subtil dans sa modernité tripartite, le jeu se révèle en réalité encore plus complexe de par l'historicité des

(24) Louis Sébastien Mercier, Tableau de Paris, "Les extrêmes se touchent », chapitre CCCXLVIII, « Le diacre Pâris », chapitre DCLXI, Paris, Mercure de France, 1994, p. 955 (Première édition, 1781-1789)

(25) Louis Sébastien Mercier, Néologie, 1801. 
situations et des courants qui traversent ces trois entités. En effet, il convient de remarquer que chaque mouvement politique, quel qu'il soit, se voit à son tour traversé par ses extrémistes et ses conservateurs, dont les postures peuvent selon la conjoncture changer. Les radicaux de la veille peuvent être considérés comme des timorés du lendemain, ou se trouvent assignés selon des catégories que leur positionnement ne peut faire évoluer.

Durant le Directoire et malgré leur compréhension de la nécessité d'une conquête légale du pouvoir par le jeu des élections, « les républicains démocrates », comme les appelait Alphonse Aulard, se voient systématiquement traités d' « exagérés » mot passe-partout pour dénoncer par un a priori négatif, toute idée quelque peu en deçà ou au-delà de la pensée des « honnêtes gens », désormais au pouvoir.

Pourtant, le principal apport de l'historiographie récente n'est pas là, mais dans la repensée profonde de la partie invisible, silencieuse et ontologiquement discrète, longtemps masquée par le problème du radicalisme, et qu'il s'agira de donner à voir : la modération politique et ses sens, posant à son tour de redoutables difficultés au moment de l'aborder en temps de révolution. Longtemps mésestimée comme posture couarde, arrangeante, plutôt faible en temps de révolution, peu inventive du point de vue idéologique, la modération mérite d'être réétudiée, comme une authentique position politique revendiquée et ayant bien plus marqué la construction de l'espace politique français que les historiens ne l'ont pensé. Dès le début de la Révolution, des hommes politiques, conscients des formes de dérive de la vie politique, désirent occuper un créneau apparemment libre, celui d'une neutralité qu'ils nomment «l'Impartialité », du nom éponyme de leur journal ${ }^{26}$. À bien lire la feuille et à bien regarder ces auteurs, cette modération est toute relative mais intéressante à étudier : elle est une position de ce que l'on appellerait une droite modérée, qui affiche son goût de l'ordre du règne de la loi et désire une modernisation contrôlée de la monarchie dans laquelle ancienne aristocratie et nouvelles élites se trouveraient au sommet de l'État. Un parti de l'ordre qui s'affirme modéré est en train de naître. Il prendra à la droite de l'échiquier divers visages selon la conjoncture. Il peut être intéressant de garder à l'esprit les thèmes

(26) Sur les impartiaux, voir Robert GRIfFITHs, Le centre perdu. Malouet et les «Monarchiens " dans la Révolution française, Grenoble, PUG, 1988, et la caricature de cette modération présentée par Camille Desmoulins dans les Révolutions de France et de Brabant, n 8, janvier 1790, " portrait des impartiaux, des Modérés, des Modérateurs, autre fois dits les Aristocrates ». 
politiques qui fédèrent ces hommes : respect du pouvoir exécutif, crainte des joutes d'assemblées dans lesquelles le public est pris à partie en position d'arbitre, construction d'une politique du bon sens n'ayant nul besoin de longs discours, s'appuyant sur la «naturalité » de la défense des intérêts des particuliers, au travers de la sanctuarisation de la propriété. Cette politique là, peu visible en temps de révolution et de radicalité ne saurait être négligée car elle va bientôt devenir celle d'une majorité silencieuse qui, pour ne pas s'exprimer, n'en existe pas moins, comme le relève un philo-monarchiste, Lezay Marnésia durant le Directoire ${ }^{27}$. Un centre est né, qui met en avant le plus souvent, en guise de sa respectabilité par rapport aux autres forces politiques, sa modération, son souci de prendre en compte plus que les citoyens, les «mitoyens », ces hommes et ces femmes d'un juste milieu, éloigné des passions orageuses. Un programme se met progressivement en place, entre 1795 et 1799. Il vise à fédérer en un centre mal défini, si ce n'est dans ce qu'il n'est pas, exprimé dans la haine à l'anarchie et à la royauté, tous les citoyens, davantage préoccupés d'organisation de l'ordre public que de construction de l'espace public.

Après le constat que la Révolution est l'invention paradoxale d'une forme de modération du politique - même les concepteurs de la Terreur n'oublient jamais de la situer, de la justifier et de la légitimer dans un juste milieu -, il faudrait ajouter que la modération, pensée comme un mode de gouvernement, s'accompagne, grâce au texte constitutionnel de l'an III, d'une rigueur pragmatique puissante dans la conquête, par les représentants des « honnêtes gens », des fonctions exécutives de l'appareil d'État, laissant de côté les questions idéologiques, sans pour autant abandonner le discours politique. Ailleurs l'hypothèse a été émise de donner à cette modération feinte, le nom "d'extrême centre" dans sa capacité à construire, au nom du repos de tous, les formes les plus rigoureuses d'utilisation de la force publique et par extension de tout le monopole de la violence d'État pour se maintenir au pouvoir ${ }^{28}$. Le flottement idéologique des girouettes, leur capacité à occuper des postes de commandement dans l'État à partir de 1795, et parfois depuis 1789, et ce jusqu'en 1815 et au-delà, affirmant toujours une modération dans leur comportement idéologique, constitue un des traits caractéristiques de cette modération...

(27) Adrien de LeZAY MARnÉSIA, De la faiblesse du gouvernement et de la nécessité pour lui de gouverner selon le vœu de la multitude, Paris, 1796.

(28) Pierre SERna, La république des girouettes, 1789-1815 et au-delà une anomalie politique : la France de l'extrême centre, Seyssel, Champ Vallon, 2005. 
radicale. Loin d'être circonscrite à la France, cette reconfiguration du champ politique se retrouve au-delà des frontières. Dès la restauration aux Provinces-unies, devenues le royaume de Hollande, il est possible de repérer ces personnages modernes revendiquant une modération idéologique dans une pratique ferme du pouvoir, fermée à toute forme de démocratisation. Le constat dressé pour l'Italie semble encore plus accablant montrant les racines profondes d'un particularisme italien, celui des majorités arrangées au nom d'un centrisme bien compris des hommes au pouvoir. Loin de constituer une rupture avec la période révolutionnaire du Triennio, ces pratiques de compromis permanents et point toujours glorieux se trouveraient déjà dans les pratiques jacobines des premiers patriotes italiens. Cependant la situation de l'Italie dans sa désunion et la convoitise des autres pays laissaient-elles un autre choix à ces représentants?

$\mathrm{Au}$ terme de ce bref survol de quelques problématiques rencontrées au moment de tenter une réflexion renouvelée sur la question de la radicalité et de la modération, il conviendrait d'adopter deux attitudes au moment d'aborder les différentes études présentées, en acceptant de chausser deux paires de lunettes pour mieux voir de près et de loin à la fois. Pour voir de près. Chaque étude s'attache à montrer combien toute génération de révolutionnaires est sensible à la question de l'extrémisme comme conséquence presque normale de la compression de liberté que chaque mouvement d'émancipation vient dénoncer, libérant par là même des forces difficilement maîtrisables. À l'historien d'être attentif afin de démêler la nécessité stratégique et verbale du positionnement des acteurs pour mieux déceler leur calcul de transformation du réel. À l'historien de saisir l'authenticité novatrice d'une pensée radicale dans ce qu'elle porte de capacité de changement, non pour le présent mais pour le futur. La Révolution serait un temps où ce qui s'énonce sous la forme de la normalité du futur ne peut que choquer ceux qui l'entendent dans le présent.... C'est pour cette raison précise que la modération en temps de révolution ne saurait être seulement étudiée sous la forme de la réaction archaïque mais comme l'antécédence des reprises en main radicales que des pouvoirs liberticides n'ont jamais manqué d'imposer dans différents processus révolutionnaires. Pour voir de loin. La toile de fond de la seconde moitié du XVIII siècle est travaillée, obsédée par l'idée du juste milieu, et l'exemple anglais le montre amplement. L'espace mental des personnes qui s'avancent vers 1789 , vont vivre et réaliser la Révolution, est sans cesse empli d'expressions, d'images qui renvoient à cette tempérance que 
Georges Benrékassa a contribué à mettre en valeur ${ }^{29}$. Tous les paradigmes valorisants portent l'idée que la maîtrise des passions, l'émergence de la raison, le travail de la critique éloignent l'homme bien pensant de tout emballement radical. Il n'est pas jusqu'à la science, dans ses paradigmes dominants qui ne conforte dans cette idée. Ainsi un Virey, bien connu des milieux scientifiques par ses entreprises éditoriales de vulgarisation, n'aura de cesse de reprendre pour la rendre parfaitement commune cette idée que l'Europe et particulièrement la France, se trouvant éloignées des extrémités chaudes et froides de la planète, se trouvent dans une position climatique et civilisationnelle idéale pour imposer leur modèle de climat et de société tempérées... notamment aux peuplades de kalmouks et d'africains entre autres.

Tout joue en faveur d'un centre invisible, sans cesse rappelé comme valeur, vertu et point cardinal, devenu référent dominant dans bien des domaines différents des sciences, de la morale et de la politique ${ }^{30}$. Ce sera le mérite de quelques uns d'avoir su regarder ni de près ni de loin, ailleurs, vers un État sans Dieu, vers une démocratisation réelle de la vie politique, vers une éducation commune, vers une société des peuples égaux, vers une société de plus grande parité, entre autres, et d'avoir fait en sorte, par leur audace tenace ou par leur habileté tactique, que des idées radicales de la fin du XVIII ${ }^{e}$ siècle devinssent la norme de la modération à la fin du $\mathrm{XIX}^{\mathrm{e}}$ siècle, entre gens civils... Une voie à poursuivre ?

Aujourd'hui, en ces temps de réduction du pacte républicain à sa plus stricte expression et de désidéologisation intense par les médias et une société toute vouée à la consommation, y a-t-il un sens à penser la politique au travers des catégories de la modération et de la radicalité ? Quelqu'un a imaginé la situation, Restif de la Bretonne en janvier 1793 :

« Je vis les hommes de 1992, lire notre histoire... je crus voir que toute l'Europe avait pris un gouvernement nouveau, mais je voyais sur les pages de l'histoire, les horribles secousses que les nations avaient éprouvées! Il me semblait entendre les lecteurs se dire entre eux : "que nous sommes heureux, de n'avoir pas vécu dans ces temps horribles, où la vie des hommes était comptée pour rien!". Un de leurs philosophes s'écriait "il faut de temps en temps de secousses, pour faire sentir aux hommes le

(29) Georges BenRÉKASSA, « Modération, modérés, modérantisme », dans Rolf REICHARDT et Hans Jürgen LusEBrinK (dir.), Hanbush Politisch-Sozialer Grundbdgriffe in Frankreich, 1680-1820, Munich, O. Oldenbourg Verlag, 1996.

(30) Voir Joseph VIREY, Histoire naturelle du genre humain, Paris, 1801, an IX. 
prix de la tranquillité"... Mais lui dit un de ses confrères, aurais-tu voulu être le secoueur ou le secoué ?... Ha ! Les beaux raisonneurs s'écria un songe-creux tapi dans un coin. Vous étiez des hommes d'il y a deux cents ans ; vous êtes composés de leurs molécules organiques : et vous êtes en paix, parce que ces molécules sont lasses d'avoir été en guerre. Vous y reviendrez, après un long repos $»^{31}$.

Pierre SERNA

IHRF/ EA 127/UMS 622.

17 rue de la Sorbonne

75231 Paris cedex 05 pierreserna@wanadoo.fr 